TRANSACTIONS OF THE

AMERICAN MATHEMATICAL SOCIETY

Volume 352, Number 11, Pages 5357-5379

S 0002-9947(00)02475-2

Article electronically published on June 13, 2000

\title{
COTORSION THEORIES AND SPLITTERS
}

\author{
RÜDIGER GÖBEL AND SAHARON SHELAH
}

\begin{abstract}
Let $R$ be a subring of the rationals. We want to investigate self splitting $R$-modules $G$ (that is $\operatorname{Ext}_{R}(G, G)=0$ ). Following Schultz, we call such modules splitters. Free modules and torsion-free cotorsion modules are classical examples of splitters. Are there others? Answering an open problem posed by Schultz, we will show that there are more splitters, in fact we are able to prescribe their endomorphism $R$-algebras with a free $R$-module structure. As a by-product we are able to solve a problem of Salce, showing that all rational cotorsion theories have enough injectives and enough projectives. This is also basic for answering the flat-cover-conjecture.
\end{abstract}

\section{INTRODUCTION}

Jutta Hausen [13] showed in her PhD-thesis, under the supervision of Reinhold Baer in 1967, that any countable, torsion-free abelian group $G$ with $\operatorname{Ext}(G, G)=0$ is free over some ring $R \subseteq \mathbb{Q}$. This interesting result, at that time motivated by studies on automorphism groups, received new support recently from investigations of cotorsion theories; see Salce [21] and Schultz [22]. Our paper will deal with Hausen's result, that is, with groups $G$ such that $\operatorname{Ext}(G, G)=0$. Following Schultz [22], we call these modules splitters. Because of their importance, also other names are in use, see the 'Dictionary' on p. 351 in Ringel [17]. These self splitting modules are also called 'stones' by Kerner [15. (in contrast to 'bricks', which refers to the case that the endomorphism ring is a division ring, see [18), exceptional modules by Rudakov [20], and Schur modules in Unger [24, see also [25]. We will stick to the name 'splitters' introduced by Schultz [22]. Our interest in splitters comes partly from their relatives, Whitehead groups, which have been investigated thoroughly over the last two decades; see results in [4] and in the more recent paper [1]. On the other hand, we are motivated in the study of splitters by open problems concerning the algebraic structure of such modules and questions on cotorsion theories related to splitters.

First we notice a big difference between Whitehead groups and splitters, one that makes splitters more attractive. In the case of Whitehead groups one of the components in Ext (_, _) is $\mathbb{Z}$, hence countable!

Received by the editors February 23, 1998 and, in revised form, June 1, 1998 and November 18, 1998.

2000 Mathematics Subject Classification. Primary 13D30, 18E40, 18G05, 20K20, 20K35, 20K40; Secondary 03C60, 18G25, 20K35, 20K40, 20K30, 13C10.

Key words and phrases. Cotorsion theories, completions, self-splitting modules, enough projectives, realizing rings as endomorphism rings of self-splitting modules. This paper is number GbSh 647 in Shelah's list of publications.

This work is supported by the project No. G-0294-081.06/93 of the German-Israeli Foundation for Scientific Research and Development. 
Before we state our main results we will discuss the connection of cotorsion theories and splitters; see $\S 2$ for more details. Cotorsion theories were introduced by Salce [21] in 1978. They are dual to the well-known classical torsion theories, replacing the crucial Hom-functor by Ext. For reasons which will be clear soon, even if we only want to know about splitters in the category of abelian groups we are bound to consider, and will restrict ourselves to, $R$-modules over subrings $R$ of the rationals $\mathbb{Q}$.

Following 21, a pair $(\mathfrak{F}, \mathfrak{C})$ of maximal classes $\mathfrak{F}, \mathfrak{C}$ of $R$-modules is a cotorsion theory if $\operatorname{Ext}(\mathfrak{F}, \mathfrak{C})=0$ in an obvious sense. Then $\mathfrak{C}$ is the cotorsion part and $\mathfrak{F}$ is the torsion-free part of this theory $(\mathfrak{F}, \mathfrak{C})$. The most important example of a cotorsion theory is the 'classical' cotorsion theory, developed in the 60 s by Harrison and many other algebraists, is the pair (torsion-free, cotorsion), see Fuchs 6]. It is easy verified that in the classical cotorsion theory the cotorsion groups $C$ come from the rationals $\mathbb{Q}$ in the sense that

$$
\operatorname{Ext}(C, \mathbb{Q})=0 \text { if and only if } C \in \mathfrak{C},
$$

so $\mathbb{Q}$ cogenerates the cotorsion theory. Cotorsion theories cogenerated by subgroups of $\mathbb{Q}$ are called rational cotorsion theories; they are well-studied, and are the main objects in Salce's paper [21]. From the homological point of view it is important to know whether rational cotorsion theories have enough projectives and injectives. Only then will we be ready to introduce cotorsion hulls! This question was raised in Salce [21] and remained open so far. We will answer it positively in Section 6 .

The answer to Salce's question is a byproduct of our study of splitters. As already indicated, the study of abelian group splitters can be reduced to torsionfree, reduced $R$-modules $(R \subseteq \mathbb{Q})$ by a "Reduction Theorem [2.5' due to Schultz [22], where $R=\operatorname{nuc} G$ is the nucleus of $G$. The nucleus of $G$ is the largest subring $R$ of $\mathbb{Q}$ such that $G$ is (canonically) an $R$-module. The first step towards the structure of splitters is Hausen's theorem, which can be slightly extended (Theorem 2.6):

Splitters of cardinality $<2^{\aleph_{0}}$ are $\aleph_{1}$-free modules over their nuclei.

The key tool of this paper can be found in Section 3. Here we will prove our Ext-Lemma 3.3 Our original proof of the Ext-Lemma was based on constructing solutions of certain systems of equations. However, following the later paper [12] we will replace this by a homological shortcut. Nevertheless homological arguments often conceal details of the structure under investigation even though the arguments may lead more quickly to the desired result. The Whitehead problem, only settled after two decades, may explain this very well; this is one reason why we also include a non-homological proof (4.12) of (2.6) as well. This will also shed light on the ExtLemma 3.3

Obviously free groups and torsion-free cotorsion groups are splitters, the first for trivial algebraic reasons the latter by completion. Before we state the Ext-Lemma we also need a definition of $n$-free-by- $1 R$-modules, and we explain its connection with classical results. Similarly to simply presented groups, $n$-free-by- 1 groups are easy represented by free generators and relations. If we want to find non-free splitters $G$, which are necessarily of cardinality $\geq \aleph_{1}$ by (2.6), one of the obstacles are "small" non-free subgroups of $G$. If $G$ has non-free countable subgroups, then by Pontryagin's theorem $G$ also has non-free subgroups of some minimal finite rank $n+1$. These groups are investigated under the name $n$-free-by- $R$-modules in Section 3 first. The name is easily explained; see Observation 3.1 and (3.2) for their elementary properties. Rational groups are the special 0 -free-by- $1 \mathbb{Z}$-modules, but 
those for $n \geq 1$ or $n=\omega$ are particular important. Such groups $G^{\prime}$ are canonically connected with certain "easy" systems of equations (3.2), and another group $G$ is called $G^{\prime}$-complete if these equations are always solvable in $G$. This observation surely is connected with ideas of type in model theory; see Prest [16]. Our crucial Ext-Lemma now reads as follows:

Ext-Lemma 3.3 Let $G^{\prime}$ be an $n$-free-by-1 $R$-module. Any torsion-free module $G$ over the nucleus $R$ is $G^{\prime}$-complete if and only if $\operatorname{Ext}\left(G^{\prime}, G\right)=0$.

As indicated, $\mathbb{Z}$-adically complete modules are $G^{\prime}$-complete modules for a suitable module $G^{\prime}$. If $\operatorname{Ext}(\mathbb{Q}, G)=0$ and $G$ is torsion-free and reduced, then $G$ is complete in the $\mathbb{Z}$-adic topology by classical results due to Kaplansky, see [6]. Hence splitting quite often implies completion. Such torsion-free modules are also cotorsion modules, hence algebraically compact. They have a nice structure theory by cardinal invariants studied by many algebraists; see Warfield 26, Ziegler 27] and work by Łoš, Kaplansky and others, see e.g. [6] 4]. Moreover, they are the cotorsion-splitters of the classical cotorsion theory. If $\mathbb{Q}$ is replaced by another rational group, this can also be seen in Salce 21. Note that the structure theory for these rational cotorsion theories in Salce [21] extends easily for $n$-free-by-1 $R$-modules.

In the first part of Section 4 we deduce classical results, like Kaplansky's theorem (above), from the Ext-Lemma. Then we clarify the new situation shown by 3.3 $G^{\prime}$-complete $R$-modules do not need to be complete in any $S$-adic topology. There are $n$-free counterexamples (where any submodule of rank $<n$ is free). Their endomorphism ring (with free additive structure) may be prescribed as well (Theorem 4.9). This shows that, in contrast to classical $\mathbb{Z}$-adically complete groups, these $G^{\prime}$-complete $R$-modules $G$ cannot be classified by any reasonable invariants. This is the case even if $G$ has a nice-looking filtration built up by copies of $G^{\prime}$ (Theorem 4.11). Some of these $n$-free examples turn out to be splitters, as shown in Section 5 , and we are able to prescribe endomorphism rings of splitters. We will see that for a given number $n>1$ there are $n$-free splitters of size $2^{\aleph_{0}}$ (and larger), which answers an open problem. These $n$-free modules are obviously cotorsion-free. Modifying our arguments, Eklof noticed that $n$-free can be replaced by $\aleph_{1}$-free if the size of the splitter is at least $\left(2^{\aleph_{0}}\right)^{+}$. Changing the construction again slightly as in [12, it is now also possible to show that there are non-free but $\aleph_{1}$-free and slender splitters of this cardinality, see 12 . Hence it should be interesting to study $\aleph_{1}$-free splitters of cardinality $\aleph_{1}$. This earlier part of Section 7 grew to an individual joint paper 11. We would like to thank Paul Eklof for pointing out a wrong argument in that first version part of Section 7, which as a consequence gave rise to 11. Here we show that these modules are free indeed in ordinary set theory (ZFC).

\section{Cotorsion theories AND SPlitTers. A SUMMARY OF KNOWN FACTS AND DEFINITIONS; SOME GENERALIZATIONS}

In 1966 S. E. Dickson [3] introduced torsion theories for abelian categories by exploiting the Hom-functor. This helped to overcome difficulties in defining torsion submodules and provided grounds for new research. Replacing formally the Hom-functor by the Ext-functor, Salce [21] developed the basic tools for a cotorsion theory, which naturally extends the 'classical cotorsion theory', the notion of cotorsion (modules) abelian groups introduced by Harrison, Nunke, Fuchs, Kaplansky 
and others, see Fuchs 6, p.232]. We will use some notation from Göbel and Prelle [10] to introduce Salce's cotorsion-theory. If $\mathfrak{X}, \mathfrak{Y}$ are two classes of abelian groups, we say that

(i) $\mathfrak{X} \perp \mathfrak{Y}$ if and only if $\operatorname{Ext}(X, Y)=0$ for all $X \in \mathfrak{X}$ and $Y \in \mathfrak{Y}$. Moreover

(ii) $\mathfrak{X}^{\perp}$ is the unique largest class of abelian groups with $\mathfrak{X} \perp \mathfrak{X}^{\perp}$, and dually

(iii) $\perp \mathfrak{X}$ is the unique largest class of abelian groups with ${ }^{\perp} \mathfrak{X} \perp \mathfrak{X}$.

The class $\mathfrak{X}^{\perp}$ is called the injective closure of $\mathfrak{X}$ and ${ }^{\perp} \mathfrak{X}$ is the projective closure of $\mathfrak{X}$, notions which are natural in view of Salce 21]. Following Salce 21] we say that a pair $(\mathfrak{F}, \mathfrak{C})$ of classes $\mathfrak{F}, \mathfrak{C}$ of abelian groups is a cotorsion theory if the following conditions hold.

(iv) $\begin{cases}(a) & \mathfrak{F} \perp \mathfrak{C} . \\ (b) & \text { The classes are maximal: } \mathfrak{C}=\mathfrak{F}^{\perp} \text { and } \mathfrak{F}={ }^{\perp} \mathfrak{C} .\end{cases}$

$\mathfrak{C}$ is the cotorsion-part and $\mathfrak{F}$ is the torsion-free class of this theory. The 'classical cotorsion theory' $\left(\mathfrak{F}_{c}, \mathfrak{C}_{c}\right)$ deals with the torsion-free part $\mathfrak{F}_{c}=$ all torsion-free groups, and the cotorsion-part $\mathfrak{C}_{c}=$ all cotorsion groups.

There are two dual ways to produce new cotorsion theories from a given class $\mathfrak{X}$ of abelian groups. We begin either with $\perp^{\perp} \mathfrak{X}$ or with $\mathfrak{X}^{\perp}$, and get, respectively,

(v) $\left\{\begin{array}{l}\mathfrak{X} \text { generates the cotorsion theory }\left({ }^{\perp} \mathfrak{X},\left({ }^{\perp} \mathfrak{X}\right)^{\perp}\right) . \\ \text { Dually, } \mathfrak{X} \text { cogenerates the cotorsion theory }\left({ }^{\perp}\left(\mathfrak{X}^{\perp}\right), \mathfrak{X}^{\perp}\right) .\end{array}\right.$

It is easy to check that the pairs in (v) are cotorsion theories and the "classical cotorsion theory' is cogenerated by the rationals $\mathbb{Q}$, i.e. $\left(\mathfrak{F}_{c}, \mathfrak{C}_{c}\right)=\left({ }^{\perp}\left(\mathbb{Q}^{\perp}\right), \mathbb{Q}^{\perp}\right)$. The crucial part is $\mathfrak{C}_{c}=\mathbb{Q}^{\perp}$, i.e., Ext $(\mathbb{Q}, G)=0$ for all cotorsion modules $G$. This well-known fact [6] is basic in $\S 3$. The well-studied classical cotorsion theory suggests a close investigation of their immediate relatives coming from subgroups of $\mathbb{Q}$ :

Suppose $S \subseteq \mathbb{Q}$ is a rank-1 group and assume that $1 \in S$ without loss of generality. The main task of Salce [21] is a detailed description of the cotorsion theory $\left(\mathfrak{F}_{S}, \mathfrak{C}_{S}\right)$ cogenerated by $S$. These cotorsion theories (for any $S \subseteq \mathbb{Q}$ ) are called rational cotorsion theories. We will make use of Salce's main theorem describing the class $S^{\perp}$ of $S$-cotorsion groups in $\left({ }^{\perp}\left(S^{\perp}\right), S^{\perp}\right)$. The following three standard definitions are needed:

(vi) $\chi_{S}=\chi(1)=\left(r_{p}\right)_{p \in \Pi}$

(where $\Pi$ is the set of primes) is the characteristic of $S$ with $p^{r_{p}}$ the maximal $p$-power dividing 1 in $S$, or $r_{p}=\infty$ if $p$ divides 1 infinitely often.

(vii) $G_{S}=\bigcap_{p \in \Pi} p^{r_{p}} G$,

where $p^{\infty} G=\bigcap_{\sigma} p^{\sigma} G$ is defined by the Ulm sequence inductively for all ordinals: $p^{\sigma+1} G=p\left(p^{\sigma} G\right)$, and $p^{\sigma} G=\bigcap_{\nu<\sigma} p^{\nu} G$ for limit ordinals $\sigma$.

(viii) If $r_{p}<\infty$, then $G_{p}^{S}=G / p^{r_{p}} G$, and $G_{p}^{S}=\dot{G}_{p}=\operatorname{Ext}\left(Z\left(p^{\infty}\right), G\right)$ if $r_{p}=\infty$.

Theorem 2.1 ([21]). If $S \subseteq \mathbb{Q}$ is as above, then $G$ is $S$-cotorsion $\Longleftrightarrow G / G_{S}$ is cotorsion $\Longleftrightarrow G / G_{S} \cong \prod_{p \in \Pi} G_{p}^{S}$.

For homological consideration it is very important to find out whether a category has enough injectives and projectives. A cotorsion theory $(\mathfrak{F}, \mathfrak{C})$ has enough injectives if and only if for all abelian groups $G$ there are $C \in \mathfrak{C}, F \in \mathfrak{F}$ and a short exact sequence

$$
0 \rightarrow G \rightarrow C \rightarrow F \rightarrow 0
$$


Dually, $(\mathfrak{F}, \mathfrak{C})$ has enough projectives if and only if there is another short exact sequence

$$
0 \rightarrow C \rightarrow F \rightarrow G \rightarrow 0 .
$$

An easy lemma reduces the question about the existence of injectives and projectives to one problem.

Lemma $2.2([21])$. A cotorsion theory $(\mathfrak{F}, \mathfrak{C})$ has enough projectives if for all free groups $A$ there are $C \in \mathfrak{C}, F \in \mathfrak{F}$ and a short exact sequence

$$
0 \rightarrow A \rightarrow C \rightarrow F \rightarrow 0 .
$$

Hence $(\mathfrak{F}, \mathfrak{C})$ has enough projectives if and only if it has enough injectives.

Classical results, widely used, show that any abelian group $A$ can be purely embedded into its cotorsion hull $A^{\bullet}$, see [6, p. 248]. Hence the classical cotorsion theory has enough injectives, and, by the above, enough projectives. Naturally, Salce [21, Problem 2, p. 31] raised the question of whether rational cotorsion theories have enough projectives (injectives). We will answer this question in the affirmative in a more general context - as a by-product of our study of splitters.

'Splitters' were introduced in Schultz 22. They also come up under different names; see the introduction. Moreover, we will see immediately that splitters are closely connected with Salce's work 21. Recall that the cotorsion class $\mathfrak{C}$ of a cotorsion theory $(\mathfrak{F}, \mathfrak{C})$ is closed under epimorphic images. Similarly, $\mathfrak{F}$ is closed under subgroups. If $F \in \mathfrak{F}, C \in \mathfrak{C}$ are the groups in the exact sequence to define enough projectives, respectively injectives, for $G$, then either $F \in \mathfrak{F} \cap \mathfrak{C}$ or $C \in \mathfrak{F} \cap \mathfrak{C}$. Hence $\mathfrak{F} \cap \mathfrak{C}$ is particular important. In the classical case this is the class of torsionfree cotorsion groups, or equivalently torsion-free algebraically compact groups, which can be classified by cardinal invariants; an extension to rational cotorsiongroups is given in Salce [21]. Elements $G$ in $\mathfrak{F} \cap \mathfrak{C}$ obviously satisfy the condition $\operatorname{Ext}(G, G)=0$, i.e. the sequence

(ix) $\quad 0 \rightarrow G \rightarrow * \rightarrow G \rightarrow 0$ always splits and $G$ is self splitting or a splitter.

We arrive at

Definition 2.3 (22]). An $R$-module $G$ over a (hereditary) commutative $\operatorname{ring} R$ is an $R$-splitter if any $R$-module sequence (ix) splits or, equivalently, $\operatorname{Ext}_{R}(G, G)=0$.

We are mainly interested in subrings $R$ of $\mathbb{Q}$. In this case, if $G$ is a torsion-free $R$-module, then

(x) $\operatorname{Ext}_{R}(G, G)=\operatorname{Ext}_{\mathbb{Z}}(G, G)$

because $\mathbb{Z}$-homomorphisms are $R$-homomorphisms of $G$, and we call $G$ a splitter if (2.3) holds. Obvious examples of splitters are the torsion-free cotorsion groups in $\mathfrak{F}_{c} \cap \mathfrak{C}_{c}$, coming from the classical cotorsion theory $\left(\mathfrak{F}_{c}, \mathfrak{C}_{c}\right)$. The other example comes from a trivial cotorsion theory

$$
(\mathfrak{F}=\text { free groups, } \mathfrak{C}=\text { all groups }),
$$

hence free groups in $\mathfrak{F} \cap \mathfrak{C}$ are splitters. In view of the countable case of (2.6) and the above, Schultz [22, Problem 4] raised the question of whether these are all splitters. We will answer this question to the negative in Section 5. However, following Schultz [22], we first reduce the problem to the torsion-free case.

In order to investigate splitters, Schultz [22] introduced the very useful notion of a nucleus of a group. 
Definition 2.4. The nucleus of a torsion-free group $G \neq 0$ is the largest subring $R=\operatorname{nuc} G$ of $\mathbb{Q}$ such that $G$ is an $R$-module. Hence $R$ is generated as a subring of $\mathbb{Q}$ by all $\frac{1}{p}$ ( $p$ any prime) for which $G$ is $p$-divisible, i.e. $p G=G$.

We will fix this notion $R=\operatorname{nuc} G$ of a nucleus of $G$ throughout this paper. The following result reduces the study of splitters among abelian groups to those which are torsion-free and reduced modules over their nuclei.

Theorem 2.5 (22]). Let $G$ be any abelian group and $G=D \oplus C$ a decomposition of $G$ into the maximal divisible subgroup $D$ and a reduced complement $C$. Moreover let $\pi$ be the set of all those primes for which $D$ has a non-trivial primary component. Then the following conditions are equivalent.

(i) $G$ is a splitter.

(ii) $\left\{\begin{array}{l}\text { (a) } C \text { is a torsion-free (reduced) splitter with } \\ p C=C \text { for all } p \in \pi . \\ \text { (b) } D \text { is not torsion and } C \text { is cotorsion. }\end{array}\right.$

For convenience of the reader we sketch the essential steps of the proof. (2.5) is based on an easy observation, see [6]:

(*) If $\operatorname{Ext}(A, B)=0, A^{\prime} \subseteq A, B^{\prime} \subseteq B$, then $\operatorname{Ext}\left(A^{\prime}, B / B^{\prime}\right)=0$ as well.

A short exact sequence

$$
0 \longrightarrow B \stackrel{\beta}{\longrightarrow} C \stackrel{\alpha}{\longrightarrow} A \rightarrow 0
$$

represents 0 in $\operatorname{Ext}(A, B)$ if and only if there is a splitting map $\gamma: A \longrightarrow C$ such that $\gamma \alpha=i d_{A}$.

(ii) $\rightarrow$ (i) is obvious, and (i) and (*) imply $\operatorname{Ext}(C, C)=0$. To prove that $C$ is torsion-free, assume to the contrary, that $C$ contains a copy $Z_{p}$ of a cyclic group of order $p$ for some prime $p \neq 1$, and consider the two cases $p C=C$ and $p C \neq C$. The case $p C=C$ is impossible because $Z\left(p^{\infty}\right)$ would be a subgroup of $C$, but $C$ is reduced. If $p C \neq C$, we find $C^{\prime} \subset C$ with $C / C^{\prime} \cong Z_{p}$. Observation (*) gives $0=\operatorname{Ext}\left(Z_{p}, C / C^{\prime}\right)=\operatorname{Ext}\left(Z_{p}, Z_{p}\right) \cong Z_{p}$, a contradiction; so $C$ is torsion-free. If the $p$-primary component $D_{p} \neq 0$, then $\operatorname{Ext}\left(Z\left(p^{\infty}\right), C\right)=0$ by $(*)$, hence $p C=C$ by [6. Theorem 52.3]. If $\mathbb{Q} \subseteq D$, then $\operatorname{Ext}(\mathbb{Q}, C)=0$ by $(*)$, and $C$ must be cotorsion.

From now on we will assume that $G$ is a torsion-free, reduced $R$-module with nuc $G=R$. A classical result due to Hausen [13] states that countable splitters $G$ are free $R$-modules indeed. This can be slightly extended to say

Theorem 2.6. If $R=\operatorname{nuc} G$ is the nucleus of the torsion-free group $G$ and $G$ is a splitter of cardinality $<2^{\aleph_{0}}$, then $G$ is an $\aleph_{1}$-free $R$-module.

Recall that $G$ is an $\aleph_{1}$-free $R$-module if any countably generated $R$-submodule is free. We will say that $G$ is $n$-free for some natural number $n$ if all the submodules of $G$ of rank $\leq n$ are free. This agrees with our notion of $n$-free-by- $1 R$-modules in Section 3. Theorem [2.6 will be important in Section 7. We will provide a quite obvious homological proof, followed later by some direct arguments leading to the same result. We believe that the direct arguments uncover what's hidden by homology! The non-homological proof is at the end of Section 4 in Corollary 4.12 .

First proof of (2.6) (the homological approach). The proof follows in two steps. It is convenient to recall $(*)$. Also we say that an $R$-submodule $E$ of an $R$-module $C$ has full rank if $C / E$ is torsion. First we claim 


\section{(a) $\left\{\begin{array}{l}\text { If } E \text { is a full rank } R \text {-submodule of an } R \text {-submodule } C \text { of finite rank, } \\ \text { both } E \text { and } G \text { have the same nucleus } R \text {, and } \operatorname{Ext}(C, G)=0 \text {, then } \\ \text { there is another full rank submodule } F \text { with } E \subseteq F \subseteq C, F / E \\ \text { finite and } \operatorname{Ext}(C / F, G)=0 . \text { Moreover, if } E \text { is a free } R \text {-module, } \\ \text { then } F \text { is free as well. }\end{array}\right.$}

Proof. We take dual groups $X^{*}=\operatorname{Hom}(X, G)$ and let

$$
0 \rightarrow E \rightarrow C \rightarrow C / E \rightarrow 0
$$

be the obvious short exact sequence. Hence

$$
E^{*} \rightarrow \operatorname{Ext}(C / E, G) \rightarrow \operatorname{Ext}(C, G)=0 .
$$

However $\left|E^{*}\right|<2^{\aleph_{0}}$, and $|\operatorname{Ext}(C / E, G)|<2^{\aleph_{0}}$ follows. The number of primes $p$ with $\operatorname{Ext}\left((C / E)_{p}, G\right) \neq 0$ must be finite, and the $p$-primary components $(C / E)_{p}$ of the torsion module $C / E$ must be finite as well. Take $E \subseteq F \subseteq C$ with $F / E=$ $\bigoplus_{p}(C / E)_{p}$, which is finite. We have $C / E=C / F \oplus F / E$ and $\operatorname{Ext}(C / E, G)=$ $\operatorname{Ext}(F / E, G)$, hence $\operatorname{Ext}(C / F, G)=0$.

If $E$ is free and $|F / E|=n$, then $F \cong n F \subseteq E$ is free.

(b) $\left\{\begin{array}{l}\text { If } \operatorname{Ext}(H, G)=0 \text { and } H, G \text { have the same nucleus } R, \\ \text { then } H \text { is an } \aleph_{1} \text {-free } R \text {-module. }\end{array}\right.$

Proof. Let $C \subseteq H$ be any $R$-submodule of finite rank. Hence $\operatorname{Ext}(C, G)=0$ by $(*)$. Clearly there is a free $R$-submodule $E \subseteq C$ of full rank. From (a) we have $E \subseteq F \subseteq C$ with $F / E$ finite, $F$ free and $\operatorname{Ext}_{R}(C / F, G)=0$. If there is $p$ with $(C / F)_{p} \neq 0$, then $p G=G$ is a contradiction for $R$, hence $C=F$ is free. Pontryagin's theorem completes the proof.

\section{The EXT-Lemma}

The main result of this section is related to a well-known observation due to Harrison and Kaplansky (see Fuchs [6, p. 247-249]). If $G$ is torsion-free and reduced, then $G$ is cotorsion if $\operatorname{Ext}(\mathbb{Q}, G)=0$, and this is the same as saying that $G$ is complete in the (Hausdorff) $\mathbb{Z}$-adic topology. Recall that $G$ is cotorsion if and only if $\operatorname{Ext}\left(G^{\prime}, G\right)=0$ for any torsion-free group $G^{\prime}$, which explains the strength of the demand $\operatorname{Ext}(\mathbb{Q}, G)=0$. How much of the completion is left over if $\mathbb{Q}$ is replaced by particular groups $G^{\prime}$ ? If $G^{\prime}$ is a torsion-free group of rank 1, that is a subgroup of $\mathbb{Q}$, this question is answered by Theorem 3.5 in Salce [21, p.21], which is basic for his rational cotorsion theories (see Section 4). Here we are interested in relatives of these rank-1 groups, which occur naturally as subgroups of torsion-free groups. We begin with an easy motivation concerning these relatives of rank- 1 groups by showing their existence as subgroups of arbitrary torsion-free groups.

Observation 3.1. (a) If $G$ is torsion-free but not $\aleph_{1}$-free over its nucleus $R$, then there is a pure $R$-submodule $G^{\prime} \subseteq G$ of minimal finite rank which is not $R$-free.

(b) If $r k G^{\prime}=n+1$ then we can find a free $R$-module $F=\bigoplus_{m \in \omega} y_{m}^{\prime \prime} R \oplus \bigoplus_{i<n} x_{i}^{\prime \prime} R$ and elements $k_{i m}, p_{m} \in R$ (the $p_{m}$ 's constitute a divisibility chain: $p_{j} d_{j m}=p_{m}$ $(j \leq m)$ for some $\left.d_{j m} \in R\right)$ with

$$
G^{\prime} \cong F / N \text { if } N=\left\langle y_{m+1}^{\prime \prime} p_{m}-y_{m}^{\prime \prime}-\sum_{i<n} x_{i}^{\prime \prime} k_{i m}: m \in \omega\right\rangle_{R} \subseteq F
$$


(c) $N=\bigoplus_{m<n}\left(y_{m+1}^{\prime \prime} p_{m}-y_{m}^{\prime \prime}-\sum_{i<n} x_{i}^{\prime \prime} k_{i m}\right) R$ is a free $R$-module.

Proof. (a) The nucleus $R$ is a PID; hence Pontryagin's theorem applies; see Fuchs [6]. There is a pure $R$-submodule $G^{\prime}$ of finite rank which is not free. Clearly we may choose $G^{\prime}$ of minimal rank $n+1$.

(b) Let $G^{\prime}=\left\langle x_{0}, \cdots, x_{n}\right\rangle_{*}$ be the $R$-module of rank $n+1$ given by (a). By the minimality of $n$ we observe that

$$
\left\langle x_{0}, \cdots, x_{n-1}\right\rangle_{R}=\bigoplus_{i<n} x_{i} R
$$

is a free, pure submodule of $G^{\prime}$. If $G^{\prime}$ has rank 1 , then this direct sum is zero. Also note that the torsion-free rank-1 module $G^{\prime} / \bigoplus_{i<n} x_{i} R \subseteq \mathbb{Q}$ is generated by $y_{m} \in G^{\prime}(m \in \omega)$ and the relations $y_{0}=x_{n}$ and $y_{m+1} p_{m} \equiv y_{m} \bmod \underset{i<n}{\bigoplus_{i}} x_{i} R$, see Fuchs [6. Vol. 2]. Hence $y_{m+1} p_{m}-y_{m} \in \bigoplus_{i<n} x_{i} R$, and there are $k_{i m} \in R(i<n)$ such that

$$
y_{m+1} p_{m}=y_{m}+\sum_{i<n} x_{i} k_{i m} .
$$

If $F$ is the free $R$-module given in Observation 3.1 (b), then

$$
y_{m}^{\prime \prime} \rightarrow y_{m} \quad, \quad x_{i}^{\prime \prime} \rightarrow x_{i} \quad(m \in \omega, i<n)
$$

is a well-defined epimorphism from $F$ onto $G^{\prime}$ with kernel $N$ as in (3.1)(b).

(c) [Note that the proof remains valid if $n$ is replaced by $\omega$. .] Consider elements $s_{m} \in R$ such that

$$
\sum_{m=0}^{k}\left(y_{m+1}^{\prime \prime} p_{m}-y_{m}^{\prime \prime}-\sum_{i<n} x_{i}^{\prime \prime} k_{i m}\right) s_{m}=0 .
$$

The coefficient of $y_{k+1}^{\prime \prime}$ is $p_{k} s_{k}=0$; hence $s_{k}=0$. Inductively we get $s_{0}=\cdots=$ $s_{k}=0$, and the sum in (c) is direct.

Observation 3.1 explains our interest in torsion-free $R$-modules of rank $n+1$, which are extensions of free $R$-modules of rank $n$ by a torsion-free $R$-module of rank 1 . We will always assume that such a module is not free, or, equivalently, that the rank-1 group is not $R$.

We are also interested in rank- 1 extensions of free $R$-modules of countable rank. More generally, let $n \leq \omega$ and define

$$
B=\bigoplus_{i<n} x_{i} R \oplus \bigoplus_{m \in \omega} y_{m} R
$$

a free $R$-module. Consider elements $p_{m}, k_{i m}, d_{j m}$ as in Observation 3.1, with $k_{i m}=$ 0 for almost all $i$ and each $m$. Let

$$
N=\left\langle y_{m+1} p_{m}-y_{m}-\sum_{i<n} x_{i} k_{i m}: m \in \omega\right\rangle_{R}
$$

be a free submodule of $B$. The quotient module $G^{\prime}=B / N$ satisfies the relations

$$
y_{m+1}^{\prime} p_{m}=y_{m}^{\prime}+\sum_{i<n} x_{i}^{\prime} k_{i m}, \quad p_{j} d_{j m}=p_{m} \quad(j \leq m \in \omega),
$$

where $y_{m}^{\prime}=y_{m}+N$ and $x_{i}^{\prime}=x_{i}+N$. 
We will use these particular almost free $R$-modules, their representation, and related 'closures' very often. Therefore we summarize:

Definition 3.2. If $n \leq \omega$ and $R$ is a subring of $\mathbb{Q}$, then using $B$ and $N$ above, we define an $n$-free-by- $1 R$-module $G^{\prime}$ as the quotient module $B / N$, or, equivalently, the module freely generated by

$$
G^{\prime}=\left\langle y_{m}^{\prime}, \bigoplus_{i<n} x_{i}^{\prime} R: \quad m \in \omega\right\rangle_{R},
$$

except for the relation

$$
y_{m+1}^{\prime} p_{m}=y_{m}^{\prime}+\sum_{i<n} x_{i}^{\prime} k_{i m} \quad(m \in \omega)
$$

with the $p_{m}$ 's a divisibility chain as above.

Moreover, if $G$ is any torsion-free $R$-module over its nucleus $R$, then we say that $G$ is $G^{\prime}$-complete if for any sequence $c_{m} \in G(m \in \omega)$ the system of equations

$$
y_{m+1} p_{m}=y_{m}+\sum_{i<n} x_{i} k_{i m}+c_{m} \quad(m \in \omega)
$$

has solutions $y_{m}, x_{i} \in G(m \in \omega, i<n)$.

As we assume that the divisibility chain of $p_{m}$ 's in Definition 3.2 defines a proper type (not $R$ ), 0-free-by-1 $R$-modules are the old-fashioned non-free, torsion-free rank-1 $R$-modules. In Corollary 4.1 we will show that $\mathbb{Q}$-completions are the wellknown $\mathbb{Z}$-adic completions. The key for this paper is the following connection between Ext and completions.

Ext-Lemma 3.3. Let $G$ be a torsion-free $R$-module over its nucleus $R$, and let $G^{\prime}$ be an $n$-free-by-1 $R$-module for some $n \leq \omega$. Then $\operatorname{Ext}\left(G^{\prime}, G\right)=0$ if and only if $G$ is $G^{\prime}$-complete.

Proof. Let $n \leq \omega$ and let $G^{\prime}=\left\langle y_{m}^{\prime}, \underset{i<n}{\bigoplus} x_{i}^{\prime} R: \quad m \in \omega\right\rangle_{R}$ be expressed as in Definition 3.2. We have a short exact sequence

$$
0 \longrightarrow N \stackrel{\sigma}{\longrightarrow} B \longrightarrow G^{\prime} \longrightarrow 0
$$

and it follows that

$$
\operatorname{Hom}\left(G^{\prime}, G\right) \longrightarrow \operatorname{Hom}(B, G) \stackrel{\sigma^{*}}{\longrightarrow} \operatorname{Hom}(N, G) \longrightarrow \operatorname{Ext}\left(G^{\prime}, G\right) \longrightarrow \operatorname{Ext}(B, G),
$$

where $\operatorname{Ext}(B, G)=0$ by the freeness of $B$. Hence

$$
\operatorname{Ext}\left(G^{\prime}, G\right)=0 \text { if and only if } \operatorname{Hom}(B, G) \stackrel{\sigma^{*}}{\longrightarrow} \operatorname{Hom}(N, G) \text { is surjective . }
$$

We claim that this is equivalent to saying that

$$
G \text { is } G^{\prime} \text {-complete. }
$$

Suppose that $\sigma^{*}$ is surjective. Given a sequence $c_{m} \in G(m \in \omega)$, we want to find solutions $e_{m}, d_{i} \in G$ such that

$$
e_{m+1} p_{m}=e_{m}+\sum_{i<n} d_{i} k_{i m}+c_{m} .
$$


Recall from Observation 3.1 (c) and the note in the proof of (c) that

$$
\begin{aligned}
N & =\left\langle y_{m+1} p_{m}-y_{m}-\sum_{i<n} x_{i} k_{i m}: m \in \omega\right\rangle_{R} \\
& =\bigoplus_{m \in \omega}\left(y_{m+1} p_{m}-y_{m}-\sum_{i<n} x_{i} k_{i m}\right) R \subseteq B .
\end{aligned}
$$

We define a homomorphism $\varphi$ on the free generators sending $\left(y_{m+1} p_{m}-y_{m}-\right.$ $\left.\sum x_{i} k_{i m}\right)$ to $c_{m}$ for all $m \in \omega$. From surjectivity of $\sigma^{*}$ we find a homomorphism $\Phi: B \longrightarrow G$ which coincides with $\varphi$ on (the free generators of) $N$. If we put $\Phi\left(y_{m}\right)=e_{m}$ and $\Phi\left(x_{i}\right)=d_{i}$, then the last displayed equation holds and $G$ is $G^{\prime}$-complete.

Conversely, let $G$ be $G^{\prime}$-complete and let

$$
0 \longrightarrow G \stackrel{\gamma}{\longrightarrow} H \stackrel{\eta}{\longrightarrow} G^{\prime} \longrightarrow 0
$$

be a short exact sequence. We want to construct the splitting map $\sigma: G^{\prime} \longrightarrow H$ satisfying $\sigma \eta=i d_{G^{\prime}}$. Following Definition 3.2 we define $\sigma$ first on the module freely generated by the $y_{m}^{\prime}, x_{i}^{\prime}$ 's, and show that $N$ is mapped to 0 ; hence $\sigma$ will be welldefined on $G^{\prime}$. Let $x_{i}^{*}, y_{m}^{*} \in H$ be preimages of $x_{i}^{\prime}, y_{m}^{\prime} \in G^{\prime}$ under $\eta$ in the short exact sequence; hence $x_{i}^{*} \eta=x_{i}(i<n)$ and $y_{m}^{*} \eta=y_{m}^{\prime}$. The relations in Definition 3.2 viewed in $H$ give elements $c_{m} \in G(m \in \omega)$ such that

$$
y_{m+1}^{*} p_{m}=y_{m}^{*}+\sum_{i<n} x_{i}^{*} k_{i m}+c_{m} \gamma .
$$

Recall that $G$ is $G^{\prime}$-complete; hence we can find $e_{m}, d_{i} \in G \quad(m \in \omega, i<n)$ such that

$$
e_{m+1} p_{m}=e_{m}+\sum_{i<n} d_{i} k_{i m}+c_{m} .
$$

Now we correct our first choice of elements, and define

$$
x_{i}^{\prime \prime} \sigma^{\prime}=x_{i}^{*}-d_{i} \gamma \quad(i<n), \quad y_{m}^{\prime \prime} \sigma^{\prime}=y_{m}^{*}-e_{m} \gamma \quad(m \in \omega),
$$

which is defined on the 'canonical' free resolution $F$ of $G^{\prime}$, generated by elements $x_{i}^{\prime \prime}, y_{m}^{\prime \prime}$.

We must show that the relations $N \subseteq F$ defining $G^{\prime}=F / N$ are mapped to 0 . An arbitrary generator of $N$ is of the form

$$
w=y_{m+1}^{\prime \prime} p_{m}-y_{m}^{\prime \prime}-\sum_{i<n} x_{i}^{\prime \prime} k_{i m} .
$$

We apply $\sigma$, and derive

$$
\begin{aligned}
w \sigma^{\prime}= & \left(y_{m+1}^{*}-e_{m+1} \gamma\right) p_{m}-\left(y_{m}^{*}-e_{m} \gamma\right)-\sum_{i<n}\left(x_{i}^{*}-d_{i} \gamma\right) k_{i m} \\
= & y_{m+1}^{*} p_{m}-\left(e_{m+1} p_{m}\right) \gamma-y_{m}^{*}+e_{m} \gamma-\sum_{i<n} x_{i}^{*} k_{i m}+\left(\sum_{i<n} d_{i} k_{i m}\right) \gamma \\
= & y_{m}^{*}+\sum_{i<n} x_{i}^{*} k_{i m}+c_{m} \gamma-e_{m} \gamma-\left(\sum_{i<n} d_{i} k_{i m}\right) \gamma-c_{m} \gamma \\
& -y_{m}^{*}+e_{m} \gamma-\sum_{i<n} x_{i}^{*} k_{i m}+\left(\sum_{i<n} d_{i} k_{i m}\right) \gamma=0 .
\end{aligned}
$$


Hence $\sigma^{\prime}$ induces $\sigma: G^{\prime} \longrightarrow H$. Obviously $\sigma \eta=i d$ on the generators of $G^{\prime}$, and hence $\sigma \eta=i d_{G^{\prime}}$ and $\operatorname{Ext}\left(G^{\prime}, G\right)=0$.

\section{Applications of the Ext-Lemma}

In this section we will give first applications of our Ext-Lemma 3.3 which are important later on. We will begin by rederiving some known results of Harrison, Kaplansky and Salce; see [6] and [21].

Let $\rho=\left(r_{n}\right)_{n \in \omega}$ be a divisibility chain of positive integers $r_{n}\left(r_{0}=1\right.$ and $r_{n} \mid r_{n+1}$, say $r_{n+1}=q_{n} r_{n}$ for all $\left.n \in \omega\right)$. If $G$ is a torsion-free abelian group, then we define

(i) $\mathbb{Z}_{(\rho)}=\left\langle 1 / r_{n}: n \in \omega\right\rangle \subseteq \mathbb{Q}$, the rational subgroup of $\mathbb{Q}$ generated by the $1 / r_{n}$ 's.

(ii) the $\rho$-topology on $G$ to be generated by the open sets $G r_{n}$ for all $n \in \omega$,

(iii) $G_{\rho}=\bigcap_{n \in \omega} G r_{n}$.

The sequence $\rho$ is essentially the characteristic of the rational group $\mathbb{Z}_{(\rho)}$. If $\rho$ runs over all prime powers different from a fixed prime $p$, then $\mathbb{Z}_{(\rho)}=\mathbb{Z}_{(p)}$, and if $r_{n}=p^{n}$ for all $n \in \omega$, then $\mathbb{Z}_{(\rho)}=\mathbb{Q}^{(p)}$. For obvious choices of $\rho$ in (ii) we obtain the $p$-adic and the $\mathbb{Z}$-adic topology, respectively. The $\rho$-topology on $G$ is Hausdorff if and only if $G_{\rho}=0$ in (iii). We say that $G$ is $\rho$-reduced. Note that $\left(G / G_{\rho}\right)_{\rho}=0$; hence $G_{\rho}$ is a radical and $G / G_{\rho}$ is $\rho$-reduced.

Recall that $G$ is $\rho$-complete if $G$ is complete in the $\rho$-topology. We now apply our Ext-Lemma.

Corollary 4.1. Let $G$ be torsion-free and $\rho$-reduced for some $\rho$. Then

$$
\operatorname{Ext}\left(\mathbb{Z}_{(\rho)}, G\right)=0 \Longleftrightarrow G \text { is complete in the } \rho \text {-topology. }
$$

Remark: If $\rho=(n !)_{n \in \omega}$, then $\mathbb{Z}_{(\rho)}=\mathbb{Q}$ and the $\rho$-topology is the $\mathbb{Z}$-topology. Then (4.1) is due to Harrison and Kaplansky; see [6. p.235]. If $\rho=\left(p^{n}\right)_{n \in \omega}$, then the $\rho$-topology is the $p$-adic topology and $G$ is $p$-adically complete by (4.1); see Salce [21, Theorem 3.5] for a general discussion.

Proof. Let $\rho$ be as above and put $y_{m}^{\prime}=1 / r_{n}$. Then

$$
y_{m+1}^{\prime} q_{n}=y_{m}^{\prime} \text { and } q_{m} r_{m}=r_{m+1},
$$

and $\mathbb{Z}_{(\rho)}$ is a 0 -free-by- $1 \mathbb{Z}$-module by (3.2). By the Ext-Lemma 3.3 we note that $\operatorname{Ext}\left(\mathbb{Z}_{(\rho)}, G\right)=0$ is equivalent to saying that $G$ is $\mathbb{Z}_{(\rho)}$-complete in the sense of Definition 3.2. Hence any sequence $c_{n} \in G$ gives rise to a solution $y_{n} \in G$ of the equations

$$
y_{n+1} q_{n}=y_{n}+c_{n}
$$

for $n \in \omega$. Using $(\rho)$ above, we see that $y_{0}=y_{1} r_{1}-c_{0}=y_{2} r_{2}-\left(c_{0}+c_{1} r_{1}\right)$, and inductively it follows that $y_{0}=y_{n} r_{n}-\sum_{i=0}^{n-1} c_{i} r_{i}$; hence $-y_{0}=\sum_{i \in \omega} c_{i} r_{i} \in G$. Any sequence $c_{n} \in G$ has a limit $\sum_{i \in \omega} c_{i} r_{i} \in G$, and $G$ is complete in the $\rho$-topology. The converse is obvious, and Corollary 4.1 is proved.

The following definition extends the notion of a splitter.

Definition 4.2. We say that the $R$-module $G$ over its nucleus $R$ is a finite-rank splitter if and only if $\operatorname{Ext}\left(G^{\prime}, G\right)=0$ for all finite rank $R$-submodules $G^{\prime}$ of $G$. 
Note that splitters are finite rank splitters. The existence of non-free but $\aleph_{1}$-free splitters mentioned in the introduction shows that the converse does not hold; see also [1], [12].

Proposition 4.3. If $U \neq 0$ is a pure subgroup of a finite-rank splitter $G$ and $p^{-1} \in \operatorname{nuc} U \backslash \operatorname{nuc} G$, then $\operatorname{Ext}\left(\mathbb{Q}^{(p)}, G\right)=0$.

Proof. Let $\bar{G}=G / p^{\omega} G$, where $p^{\omega} G=\bigcap_{n \in \omega} G p^{n}$, and note that $p^{\omega} \bar{G}$ is $p$-divisible and $\bar{G}$ is $p$-reduced. Let

$$
G^{\prime}=\left\langle y_{m}: m \in \omega, y_{m+1} p=y_{m}\right\rangle \subseteq U ;
$$

then $G^{\prime} \cong \mathbb{Q}^{(p)}$. If $G$ is a finite-rank splitter, then $\operatorname{Ext}\left(G^{\prime}, \bar{G}\right)=0$ and by (4.1) $\bar{G}$ is a complete module over the $p$-adic integers $J_{p}$. Hence $\bar{G}$ is cotorsion, see Fuchs 6, p. 163]. Now it is easy to see that $\operatorname{Ext}\left(\mathbb{Q}^{(p)}, G\right)=0$ as well; note that $\bar{G}$ is $q$-divisible by all primes $q \neq p$ (e.g. apply Salce [21, p. 21]), see Theorem [2.1.

We have the immediate

Corollary 4.4. If $G$ is a finite-rank splitter which is $p$-reduced for all primes $p$ and $0 \neq U$ is pure in $G$, then nuc $U=\operatorname{nuc} G$.

Proof. If $p^{-1} \in \operatorname{nuc} U \backslash \operatorname{nuc} G$, then $\operatorname{Ext}\left(\mathbb{Q}^{(p)}, G\right)=0$ by $\underline{4.3}$ ), and $G$ is a $J_{p}$-module, which contradicts that $G$ is $q$-reduced for $q \neq p$.

Remark 4.5. Note that $G=J_{p} \oplus J_{q}(p \neq q)$ is a splitter with nuc $J_{p} \neq \operatorname{nuc} G$. Hence the hypothesis in (4.4) cannot be dropped.

Corollary 4.1 might support the conjecture that a similar completeness, e.g. for a different topology, would follow for (non-free) $n$-free-by-1 groups in place of $\mathbb{Z}_{(\rho)} \subseteq \mathbb{Q}$.

The following Theorem 4.8 however, shows that such a conjecture fails dramatically. The same example and modifications will serve a different purpose later on as well. We begin with a definition which generalizes $G^{\prime}$-complete modules, in order to deduce a result on rational cotorsion theories in Section 6.

Definition 4.6. Let $\Phi$ be a set of finite-rank-free-by- $R$-modules and $\mathfrak{F}$ the class of all free $R$-modules over some proper subring $R \subset \mathbb{Q}$.

(a) An $R$-module $L$ is $\Phi$-complete if and only if $\operatorname{Ext}\left(G^{\prime}, L\right)=0$ for all $G^{\prime} \in \Phi$.

(b) An $R$-module $G$ is $\Phi$-represented if $G$ can be written as $\bigcup_{\alpha<\lambda} G_{\alpha}$, a union of an ascending continuous chain of $R$-submodules $G_{\alpha}$ with $G_{0}=0$ and $G_{\alpha+1} / G_{\alpha} \in$ $\Phi \cup \mathfrak{F}$ for any $\alpha \in \lambda$.

Remark 4.7. If $G$ is a torsion-free $R$-module, then we can find $\Phi$ such that $G$ is $\Phi$-represented: We define $\Phi$ and $\left\{G_{\alpha}: \alpha<\alpha^{*}\right\}$ inductively. If $G / G_{\alpha}$ is $\aleph_{1^{-}}$ free, then choose any countable extension $G_{\alpha+1}$ of $G_{\alpha}$ which is pure in $G$ such that $G_{\alpha+1} / G_{\alpha}$ is free over $R$. Otherwise, there is a non-free, torsion-free, pure submodule $G_{\alpha+1} / G_{\alpha} \subseteq G / G_{\alpha}$ of minimal rank $n$ by Pontryagin's theorem. From Observation 3.1 we infer that $G_{\alpha+1} / G_{\alpha}$ is an $n$-free-by-1 $R$-module which we add to $\Phi$. In case of limit ordinals, we just take unions to define the next member of the chain.

Theorem 4.8. Let $\Phi$ be a non-empty set of finite-rank-free-by-1 $R$-modules for some proper subring $R$ of $\mathbb{Q}$, and let $\kappa>|\Phi|$ be some infinite, regular cardinal with 
$\kappa=\kappa^{\aleph_{0}}$. Then there exists a torsion-free $\Phi$-complete, $\Phi$-represented $R$-module $G$ of rank $\kappa$. If all modules in $\Phi$ have rank at least $n+1$, then any $R$-submodule of rank $\leq n$ in $G$ is free.

Remark. The last theorem is of particular interest if $\Phi$ is a singleton. If the module in $\Phi$ does not have rank 1 , then $G$ in Theorem 4.8 has many free, pure submodules of rank at least 1 , which shows that $G$ cannot be complete in its $p$-adic topology or any of its natural generalizations.

Proof. We begin with a set $G$ of cardinality $\kappa$, only used for enumerating all $\omega$-tuples of elements in $G$ to ensure that the final module $G$ has solutions to all required equations linked to $\Phi$. Alternatively we can enumerate all $\omega$-tuples of elements in $G_{\alpha}$ of each submodule (with repetitions) while doing the transfinite construction of $G$.

Let $G_{0}=0$, and let $G_{1}=\bigoplus_{i \in \kappa} e_{i} R$ be a free $R$-module of rank $\kappa$, and choose a set $G \supset G_{1}$ with $\left|G \backslash G_{1}\right|=\kappa$ from which we will pick an ascending continuous chain $G_{\alpha}$ of submodules $(\alpha<\kappa)$ with $\left|G \backslash G_{\alpha}\right|=\kappa$. We also choose an enumeration $\bar{c}^{\alpha}=\left(c_{n}^{\alpha}: n \in \omega\right)$ of $\omega$-tuples of $G(\alpha<\kappa)$ such that each $\bar{c} \in G^{\omega}$ appears $\kappa$ times, i.e. $\left|\left\{\alpha \in \kappa: \bar{c}^{\alpha}=\bar{c}\right\}\right|=\kappa$.

Similarly we choose an enumeration of $\Phi$ by $X_{\alpha}(\alpha \in \kappa)$ with $\kappa$-repetitions.

If $G_{\alpha}$ is constructed, then we distinguish two cases for constructing $G_{\alpha+1}$.

Case 1: There is a $c_{n}^{\alpha} \in G \backslash G_{\alpha}$ for some $n \in \omega$. We set $G_{\alpha+1}=G_{\alpha} \oplus R$.

Case 2: If $c_{n}^{\alpha} \in G_{\alpha}$ for all $n \in \omega$, then we apply Lemma 3.3. There is an extension $G_{\alpha+1} \supset G_{\alpha}$ such that

$$
\begin{aligned}
G_{\alpha+1} / G_{\alpha}=\left\langle y_{m}^{\alpha}+G_{\alpha}, x_{i}^{\alpha}+G_{\alpha}: i<n\right\rangle & \cong X_{\alpha} \\
& \text { with } y_{m+1}^{\alpha} p_{m}=y_{m}^{\alpha}+\sum_{i<n} x_{i}^{\alpha} k_{i m}+c_{m}^{\alpha}
\end{aligned}
$$

where $p_{m}^{\alpha}, k_{i m}^{\alpha} \in R(m \in \omega)$ come from (3.2) applied to $X_{\alpha}$. Hence

$$
G_{\alpha+1}=\left\langle G_{\alpha}, y_{m}^{\alpha}, x_{i}^{\alpha}: i<n, m \in \omega\right\rangle .
$$

Finally, $G=\bigcup_{\alpha \in \kappa} G_{\alpha}$ is the union of the continuous chain $\left\{G_{\alpha}: \alpha \in \kappa\right\}$, and $G$ is torsion-free of rank $\kappa$.

If $\bar{c}=\left(c_{m}\right) \in G^{\omega}$, then there is some $\beta<\kappa$ with $\bar{c} \in G_{\beta}^{\omega}$ because $c f \kappa>\aleph_{0}$. By enumeration we also find $\alpha>\beta$ such that $\bar{c}=\bar{c}^{\alpha}$; the desired solution for (3.2) is in $G_{\alpha+1}$ by construction of $G_{\alpha+1}$. Hence $G$ is $G^{\prime}$-complete and $\operatorname{Ext}\left(G^{\prime}, G\right)=0$ by (3.3).

Next we assume that all modules in $\Phi$ have rank at least $n+1$. It remains to show that any pure submodule $F$ of rank $\leq n$ is free. If this is shown for $\operatorname{rk} F=k<n$, then let $F$ be of rank $k+1$. We can choose $\beta \in \kappa$ minimal with $F \subseteq G_{\beta}$. If $\beta=0$, then $F \subseteq \subseteq_{*} G_{0}$, which is free, and our claim holds. If $\beta>0$, then $\beta$ cannot be a limit ordinal; hence $\beta=\alpha+1$.

If $\alpha$ belongs to Case 1 , then $F \subseteq_{*} G_{\alpha} \oplus R$ and $F \nsubseteq G_{\alpha}$. We see that $F /\left(G_{\alpha} \cap F\right) \cong$ $F+G_{\alpha} / G_{\alpha} \cong R$ and $\left(G_{\alpha} \cap F\right) \oplus g R=F$ with $\operatorname{rk}\left(G_{\alpha} \cap F\right) \leq k$. The induction hypothesis for $G_{\alpha} \cap F \subseteq_{*} G_{\alpha}$ completes this case.

If $\alpha$ belongs to Case 2, we argue similarly: $F$ is a pure submodule of

$$
G_{\alpha+1}=\left\langle G_{\alpha}, y_{m}^{\alpha}, x_{i}^{\alpha}: i<n, m \in \omega\right\rangle
$$


and $F \nsubseteq G_{\alpha}$. We see that

$$
F /\left(G_{\alpha} \cap F\right) \cong\left(F+G_{\alpha}\right) / G_{\alpha} \subseteq G_{\alpha+1} / G_{\alpha} \cong G^{\prime} .
$$

Obviously $\operatorname{rk}\left(F /\left(G_{\alpha} \cap F\right)\right) \leq k<n$, and any subgroup of rank $<n$ of $G^{\prime}$ is free; hence $F /\left(G_{\alpha} \cap F\right)$ is free and splits $\left(G_{\alpha} \cap F\right) \oplus F^{\prime}=F$, where $\operatorname{rk} F^{\prime} \geq 1$. Induction completes this case as well.

Finally we modify the proof of Theorem 4.8 to get

Theorem 4.9. Let $A$ be a ring with free additive group $A^{+}$of cardinality $|A|<\kappa$ for some regular cardinal $\kappa=\kappa^{\aleph_{0}}$, and let $G^{\prime}$ be an $n$-free-by-1 abelian group for some $n>0$. Then there exists an abelian torsion-free $G^{\prime}$-complete group $G$ of rank $\kappa$ such that End $G \cong A$.

Proof. We adopt the construction in the proof of Theorem 4.8 for $\Phi=\left\{G^{\prime}\right\}$, adding intermediate steps from constructions of abelian groups with prescribed endomorphism rings, based on the stationary version of Shelah's black box (see Franzen and Göbel [5]).

We enumerate the traps $\tau_{\alpha}\left(\alpha \in \lambda^{*}\right)$ of the black box such that the construction of the module depends on the norm $\left|\tau_{\alpha}\right|$ of the trap $\tau_{\alpha}$, which takes values in $\kappa$. Let $S_{1}, S_{2}$ be two disjoint stationary subsets of $\kappa$, and assume that the enumeration of $\omega$-tuples $\bar{c}^{\alpha}$ in (4.8) uses only $\alpha \in S_{1}$ as indexing set rather than $\kappa$. We assume the reader to be familiar with the construction in [2] or in [5]. The modifications will be quite obvious.

Let $G_{0}=\bigoplus_{i \in \kappa} e_{i} A$ and note that $U=\widehat{G}_{0}$, the $p$-adic completion of $G_{0}$, provides enough space to carry out the proof given in (4.8). Moreover, $\left|U^{\omega}\right|=|U|^{\aleph_{0}}=$ $\left|G_{0}\right|^{\aleph_{0}}=\kappa^{\aleph_{0}}=\kappa$, and the mentioned enumeration of $\omega$-tuples is settled. We consider $G^{\prime \prime}=G^{\prime} \otimes A$, which is the direct sum of $\operatorname{rk} A$ copies of $G^{\prime}$. If $\beta$ is a limit ordinal and all $A$-modules $G_{\alpha}(\alpha<\beta)$ are constructed, then we take $G_{\beta}=\bigcup_{\alpha<\beta} G_{\alpha}$. If $\beta=\alpha+1$, then we distinguish three main cases.

Case I. If $\left|\tau_{\alpha}\right| \in \kappa \backslash\left(S_{1} \cup S_{2}\right)$, then let

$$
G_{\alpha+1}=\left(G_{\alpha} \oplus e A\right)_{*} \subseteq U
$$

for some suitable $e \in U$.

Case II. If $\left|\tau_{\alpha}\right| \in S_{1}$, then either $\bar{c}^{\alpha} \in G_{\alpha}^{\omega}$ or not. If $c_{n}^{\alpha} \notin G_{\alpha}$ for some $n$, then we apply Case I. Otherwise let $G_{\alpha+1}^{\bullet}$ be an extension of $G_{\alpha}$ in $U$ such that $G_{\alpha+1} / G_{\alpha} \cong G^{\prime \prime}$ with "solutions" $x_{i}^{\alpha}, y_{m}^{\alpha} \in G_{\alpha+1}$ of

$$
y_{m+1}^{\alpha} p_{m}=y_{m}^{\alpha}+\sum_{i<n} x_{i}^{\alpha} k_{i m}+c_{m}^{\alpha}(m \in \omega)
$$

as in (4.8). Then we take $G_{\alpha+1}=\left(G_{\alpha+1}^{\bullet}\right)_{*} \subseteq U$.

Case III. If $\left|\tau_{\alpha}\right| \in S_{2}$, then we follow [2] or [5]. Either the trap $\tau_{\alpha}$ is of no interest (the trap does not determine a partial homomorphism or the partial homomorphism is scalar multiplication by some $a \in A$ ), in which case we apply Case I; or $\alpha$ provides an unwanted partial homomorphism $\varphi_{\alpha}$. In this case we let $G_{\alpha+1}=\left\langle G_{\alpha}, e A\right\rangle_{*}$ for some suitable $e \in U$ with $e \varphi_{\alpha} \notin G_{\alpha+1}$. This can be arranged in such a way that the support of $e$ is almost disjoint from $G_{\alpha}$ - see [2] for elements with branch-like support. Standard arguments and the proof of (4.8) ensure Theorem 4.9

Combining (4.8) and (4.9) with Lemma 3.3, we have the following. 
Corollary 4.10. Let $G^{\prime}$ be an $n$-free-by-1 group and $\kappa=\kappa^{\aleph_{0}}$ some infinite, regular cardinal. Then the following hold.

(a) There exists a torsion-free abelian p-reduced group $G$ of rank $\kappa$ with $\operatorname{Ext}\left(G^{\prime}, G\right)=0$ which is not complete in its p-adic topology.

(b) If $A$ is any ring with free additive group $A^{+}$of rank $<\kappa$, then there exists a family of $2^{\kappa}$ non-isomorphic torsion-free A-modules $G$ of rank $\kappa$ with End $G=A$ and $\operatorname{Ext}\left(G^{\prime}, G\right)=0$.

(c) There exists a family of $2^{\kappa}$ indecomposable, pairwise non-isomorphic abelian groups $G$ of rank $\kappa$ such that $\operatorname{Ext}\left(G^{\prime}, G\right)=0$.

Proof. (c) follows from (b) for $A=\mathbb{Z}$. (a) and (b) follow from the preceding results.

For applications in Section 5 we note that a modification of the proof of (4.9) leads to

Theorem 4.11. Let $A$ be a ring with free additive group $A^{+}$of rank $<\kappa$ for some regular cardinal $\kappa=\kappa^{\aleph_{0}}$, and let $G^{\prime}$ be an $n$-free-by-1 group for some $n>$ 0 . Then there exists a torsion-free $G^{\prime}$-complete A-module $G=\bigcup_{\alpha \in \kappa} G_{\alpha}$ with ascending, continuous chain $\left\{G_{\alpha}: \alpha \in \kappa\right\}$ of pure A-submodules $G_{\alpha}$ such that $G_{0}=\bigoplus_{i \in \kappa} e_{i} A, G_{\alpha+1} / G_{\alpha}$, is isomorphic either to $A$ or to $G^{\prime} \otimes A=\bigoplus_{r k A} G^{\prime}$, and End $G=A$.

Proof. The only relevant change in the proof of (4.9) is in Case III (Case I is similar but simpler). Note that we can choose (besides $e=e_{0} \in U$ ) additional elements $e_{1}, \ldots, e_{n-1} A$-independent modulo $G_{\alpha}$ such that

$$
e \varphi_{\alpha} \notin\left\langle G_{\alpha}, e_{0} A, \ldots, e_{n-1} A ; y_{m} A: m \in \omega\right\rangle=: G_{\alpha+1},
$$

where

$$
y_{m+1} p_{m} \equiv y_{m}+\sum_{i<n} e_{i} k_{i m} \quad \bmod G_{\alpha}(m \in \omega) .
$$

Hence $G_{\alpha+1} / G_{\alpha} \cong G^{\prime} \otimes A$, and we proceed as before.

We close this section with a direct proof of (2.6), as promised above.

Corollary 4.12. If $G$ is reduced and torsion-free of cardinality $<2^{\aleph_{0}}$ but not $\aleph_{1}$ free, then $G$ is not a splitter.

Proof. Suppose $G$ is a splitter, hence $\operatorname{Ext}(G, G)=0$, and let $R=\operatorname{nuc} G$. By Pontryagin's theorem there is an $R$-submodule $G^{\prime}$ of $G$ of minimal finite rank, say $n$, which is not free. Let $G^{\prime}=\left\langle y_{m}, \bigoplus_{i<n} x_{i} R\right\rangle_{R}$ and note that

$$
y_{m+1} p_{m}=y_{m}+\sum_{i<n} x_{i} k_{i m}(m \in \omega)
$$

holds as shown in (3.1), (3.2). If $n \geq 1$, then $x_{0}$ exists and is pure in $G^{\prime}$. Clearly $G^{\prime} / \bigoplus_{i<n} x_{i} R$ has type $\left(p_{m}\right)_{m \in \omega}$ and is not $R$. In this case let $c_{m}=x_{0}$, and we note that $p_{m} x=c_{m}$ has no solutions in $G^{\prime}$. If $n=0$, then nuc $G=R$ forces $p_{m} G \neq G$ and the existence of $c_{m} \in G(m \in \omega)$ such that $p_{m} x=c_{m}$ has no solution for all $m \in \omega$. We will use these elements 
(i) $\quad c_{m} \in G$ with no solution $x_{m} \in G$ for $p_{m} x_{m}=c_{m}(m \in \omega)$ to construct a non-trivial element of $\operatorname{Ext}\left(G^{\prime}, G\right)$. If $v \in \omega_{\omega}$, then let

$$
F=\bigoplus_{m \in \omega} y_{m}^{\prime \prime} R \oplus \bigoplus_{i<n} x_{i}^{\prime \prime} R \oplus G
$$

and

$$
N_{v}=\left\langle y_{m+1}^{\prime \prime} p_{m}-y_{m}^{\prime \prime}-\sum_{i<n} x_{i}^{\prime \prime} k_{i m}-c_{m} v(m): m \in \omega\right\rangle_{R} .
$$

If $H_{v}=F / N_{v}, \gamma: G \rightarrow H_{v} \quad\left(g \rightarrow g+N_{v}\right)$, then $0 \longrightarrow G \stackrel{\gamma}{\longrightarrow} H_{v}$ as in (3.3), and if $y_{m}^{\prime \prime}+N_{v}=y_{m}^{\prime}, x_{i}^{\prime}=x_{i}^{\prime \prime}+N_{v}$, then $\left(x_{i}^{\prime} \rightarrow x_{i}\right),\left(y_{m}^{\prime} \rightarrow y_{m}\right)$ induces an endomorphism $\eta_{v}: H_{v} \rightarrow G^{\prime}$ such that

(ii) $\quad 0 \longrightarrow G \stackrel{\gamma}{\longrightarrow} H_{v} \stackrel{\eta_{v}}{\longrightarrow} G^{\prime} \rightarrow 0$ is exact.

As $\operatorname{Ext}\left(G^{\prime}, G\right)=0,(i i)$ has a splitting map $\sigma_{v}: G^{\prime} \rightarrow H_{v}$ such that $\sigma_{v} \eta_{v}=i d_{G^{\prime}}$. If $e_{m}^{v}=y_{m}^{\prime}-y_{m} \sigma_{v}$ and $d_{i}^{v}=x_{i}^{\prime}-x_{i} \sigma_{v}$, then $e_{m}^{v}, d_{i}^{v} \in$ ker $\eta_{v}$ and there are $e_{m}^{v}, d_{i}^{v} \in G$ with $e_{m}^{v} \gamma=e_{m}^{\prime}, d_{i}^{v} \gamma=d_{i}^{\prime}$. The obvious relations in $G^{\prime}$ and $H_{v}$ produce (by subtraction) new relations

$$
e_{m+1}^{v} p_{m}=e_{m}^{v}+\sum_{i<n} d_{i}^{v} k_{i m}+c_{m} \gamma v(m) \quad\left(v \in{ }^{\omega} \omega\right) .
$$

Recall that $|G|<2^{\aleph_{0}}=\left|{ }^{\omega} \omega\right|$. By a pigeon-hole argument there are $v \neq w \in{ }^{\omega} \omega$ with $d_{i}^{v}=d_{i}^{w}$ for all $i<n$; then $t$ is is defined to be the branch point of $v$ and $w$, and we may assume $t>n$. Note that $v(m)=w(m)$ for all $m<t$, and $v(t)-w(t)=1$ without loss of generality; moreover, $e_{m}^{v}=e_{m}^{w}$ for all $m<t$. We subtract the two sets of relations for $v$ and $w$ respectively and get $\left(e_{n+1}^{v}-e_{n+1}^{w}\right) p_{t}=c_{t} \gamma$. As $\gamma$ is a pure embedding, the last equation contradicts our choice $(i)$ of $c_{t}$.

\section{SplitTers Which ARE NEITHER FREE NOR COTORSION}

In this section we want to answer Schultz's [22, Problem 4] in the negative by providing a list of splitters in ZFC which are neither free over their nuclei nor cotorsion. Our Examples 5.5 also show that there is no hope of classifying splitters, because any prescribed $R$-algebra $A$ which is free as a $R$-module, $R$ a proper subring of $\mathbb{Q}$, is an endomorphism algebra End $G \cong A$ of some splitter $G$ with nucleus $R$. Hence all kinds of nasty decompositions may occur, Kaplansky's test problems are violated, etc. and see Corner, Göbel [2]. We begin with

Remark 5.1. By the Ext-Lemma 3.3 we see that $L$ in (4.6) is $\Phi$-complete if and only if all systems of equations $\left(G^{\prime}\right)$ related to $G^{\prime} \in \Phi$ by (3.2) have solutions in $G$.

Theorem 5.2. If $L$ is $\Phi$-complete and $G$ is $\Phi$-represented over the same nucleus, then $\operatorname{Ext}(G, L)=0$.

Remark 5.3. We note that $L$ and $G$ must have the same nucleus $R$, which follows by trivial modification of Corollary 4.4

Proof of 5.2. Suppose $L$ and $G$ are $R$-modules as indicated and $G=\bigcup_{\alpha<\lambda} G_{\alpha}$ is the union of an ascending, continuous chain of $R$-modules over the nucleus $R$ with $G_{\alpha+1} / G_{\alpha} \in \Phi$ or $G_{\alpha+1} / G_{\alpha}$ free respectively.

We must show that any sequence

$$
0 \longrightarrow L \longrightarrow H \stackrel{\sigma}{\longrightarrow} G \longrightarrow 0
$$

splits. 
We must find a splitting map $\eta: G \longrightarrow H$ with $\eta \sigma=i d_{G}$. If $H_{\alpha}=G_{\alpha} \sigma^{-1} \subseteq H$, then $H=\bigcup_{\alpha<\lambda} H_{\alpha}$ and we construct $\eta$ by induction on $\alpha$, choosing an ascending continuous chain $\eta_{\alpha}: G_{\alpha} \longrightarrow H_{\alpha}$ of splitting maps $\eta_{\alpha} \sigma_{\alpha}=i d_{G_{\alpha}}$ for $\sigma_{\alpha}=\sigma\left\lceil H_{\alpha}\right.$. Hence $\eta=\bigcup_{\alpha<\lambda} \eta_{\alpha}$ is as required. If $\eta_{\alpha}: G_{\alpha} \longrightarrow H_{\alpha}$ with $\eta_{\alpha} \sigma_{\alpha}=i d_{G_{\alpha}}$ is given, we must find $\eta_{\alpha+1} \supset \eta_{\alpha}$ with $\eta_{\alpha+1} \sigma_{\alpha+1}=i d_{G_{\alpha+1}}$ for any $\alpha<\lambda$. If $G_{\alpha+1} / G_{\alpha}$ is free, then $G_{\alpha+1}=C_{\alpha} \oplus G_{\alpha}$ and $C_{\alpha}$ is a free $R$-module. It is easy to define $\eta_{\alpha+1} \uparrow C_{\alpha}$ from $\sigma_{\alpha+1}$; hence $\eta_{\alpha+1}$ is given component-wise. If $G_{\alpha+1} / G_{\alpha} \in \Phi$, then we may assume that $G_{\alpha+1} / G_{\alpha}$ is given by (3.2).

(ii) $\left\langle y_{m}^{\prime}, \bigoplus_{i<n} x_{i}^{\prime} R: y_{m+1}^{\prime} p_{m}=y_{m}^{\prime}+\sum_{i<n} x_{i}^{\prime} k_{i m}, m \in \omega\right\rangle_{R}$.

We take preimages $y_{m}, x_{i} \in G_{\alpha+1}$ of $y_{m}^{\prime}, x_{i}^{\prime}(m \in \omega, i<n)$ modulo $G_{\alpha}$. Note that $\sigma_{\alpha+1}: H_{\alpha+1} \longrightarrow G_{\alpha+1}$ is onto; hence we can take preimages $y_{m}^{\prime \prime}, x_{m}^{\prime \prime} \in H_{\alpha+1}$ under $\sigma_{\alpha+1}$.

$$
\text { (iii) }\left\{\begin{array}{lll}
y_{m}^{\prime \prime} \sigma=y_{m}^{\prime \prime} \sigma_{\alpha+1}=y_{m} & \text { and } \quad x_{i}^{\prime \prime} \sigma=x_{i}^{\prime \prime} \sigma_{\alpha+1}=x_{i}, \\
y_{m}+G_{\alpha}=y_{m}^{\prime} & \text { and } \quad x_{i}+G_{\alpha}=x_{i}^{\prime} \quad(m \in \omega, i<n) .
\end{array}\right.
$$

We want to find certain corrections $d_{i} \in L$ and $e_{m} \in L$ and define a preliminary map $\eta_{\alpha+1}: G_{\alpha+1} \longrightarrow \widetilde{H}_{\alpha+1}$, where $H_{\alpha+1} \subseteq \widetilde{H}_{\alpha+1}=H_{\alpha+1} \otimes \mathbb{Q}$ denotes the divisible hull of $H_{\alpha+1}$. Note that $H$ is torsion-free as an extension of torsion-free groups by $(i)$; hence $H_{\alpha+1}$ is torsion-free and the last inclusion holds. We require $\eta_{\alpha+1}\left\lceil G_{\alpha}=\eta_{\alpha}\right.$ and set

(iv) $\quad x_{i} \eta_{\alpha+1}=x_{i}^{\prime \prime}+d_{i}$ and $y_{m} \eta_{\alpha+1}=y_{m}^{\prime \prime}+e_{m} \quad(i<n, m \in \omega)$.

The mapping $\eta_{\alpha+1}$ will be a well-defined homomorphism on $G_{\alpha+1}$ if the new relations are preserved. They come from (ii) and are of the form

(v) $y_{m+1} p_{m}=y_{m}+\sum_{i<n} x_{i} k_{i m}+c_{m}^{\alpha}$ for some $c_{m}^{\alpha} \in G_{\alpha}, m \in \omega$.

Our preliminary map $\eta_{\alpha+1}$ takes these equations to $H$; by (iv) we can apply $\eta_{\alpha+1}$ to $(v)$ and get

(vi) $\quad y_{m+1}^{\prime \prime} p_{m}+e_{m+1} p_{m}=y_{m}^{\prime \prime}+\sum_{i<n} x_{i}^{\prime \prime} k_{i m}+e_{m}+\sum_{i<n} d_{i} k_{i m}+c_{m}^{\alpha} \eta_{\alpha}$

Hence $\eta_{\alpha+1}$ exists if we find solutions $e_{m}, d_{i}$ of $(v i)$.

Put $c_{m}=-y_{m+1}^{\prime \prime} p_{m}+y_{m}^{\prime \prime}+\sum_{i<n} x_{i}^{\prime \prime} k_{i m}+c_{m}^{\alpha} \eta_{\alpha}$, note that $\eta_{\alpha} \sigma_{\alpha}=i d_{G_{\alpha}}$, and calculate with $(i i i)$ and $(v)$ :

$$
c_{m} \sigma=y_{m+1} p_{m}-y_{m}-\sum_{i<n} x_{i} k_{i m}-c_{m}^{\alpha} .
$$

Hence $c_{m} \in \operatorname{ker} \sigma=L$ for all $m \in \omega$ by $(v)$. The module $L$ is $\Phi$-complete, and in particular $\operatorname{Ext}\left(G_{\alpha+1} / G_{\alpha}, L\right)=0$. By the Ext-Lemma 3.3 we can find actual elements $d_{i}, e_{m} \in L$ such that

$$
e_{m+1} p_{m}=e_{m}+\sum_{i<n} d_{i} k_{i m}+c_{m} \quad(m \in \omega) .
$$

Subtracting from $(v i)$, we obtain

(vii) $y_{m+1}^{\prime \prime} p_{m}=y_{m}^{\prime \prime}+\sum_{i<n} x_{i}^{\prime \prime} k_{i m}-c_{m}+c_{m}^{\alpha} \eta_{\alpha}$.

Our definition of $c_{m}$ above shows that (vii) holds. We have seen that $\eta_{\alpha}$ can be extended to $\eta_{\alpha+1}$ by $(i v)$ for suitable elements $d_{i}, e_{m}$. Hence $\eta$ exists and is the derived splitting map of $(i)$ by $\eta \sigma=\bigcup_{\alpha} \eta_{\alpha} \sigma_{\alpha}=\bigcup_{\alpha} i d_{G_{\alpha}}=i d_{G}$. 
Theorem 5.2 has two immediate consequences.

Corollary 5.4. If $\Phi$ is a set of finite-rank-free-by-1 R-modules and $G$ is a $\Phi$ complete, $\Phi$-represented $R$-module, then $G$ is a splitter with nucleus $R$.

Proof. Put $L=G$ and apply (5.2).

If $\Phi=\left\{G^{\prime}\right\}$ is a singleton and $G^{\prime}$ is any n-free-by- 1 abelian group for some $n>1$, then Theorem 4.11 applies. Corollary 5.4 provides a proper class of nasty examples.

Example 5.5. If $\kappa=\kappa^{\aleph_{0}}$ is any cardinal and $n$ is any integer $>1$ and $A$ is a ring of cardinality $<\kappa$ with free additive structure $A^{+}$, then there exists a splitter $G$ with End $G \cong A$ such that all its subgroups of rank $<n$ are free.

Remark 5.6. (a) We also may prescribe the nucleus $R \subset \mathbb{Q}$ of the splitter $G$ in (5.5), replacing the ring $A$ by an $R$-algebra $A$ with free $R$-module structure $A_{R}$.

(b) Any example (5.5) is a splitter but neither free nor cotorsion; hence (5.5) is a negative answer to Problem 4 in Schultz [22 p.11]. The examples exist in $Z F C$.

Problem 2 in Schultz 22 is the following question. If $G$ is a reduced torsion-free splitter with nucleus $R$ such that $G$ has no countable homomorphic image with nucleus $R$, is $G$ cotorsion? The construction of $G$ in Theorem 4.11 can be modified: Let $X$ be the direct sum of all countable torsion-free reduced abelian groups with nucleus $\mathbb{Z}$, and note that $X$ is cotorsion-free with $|X|=2^{\aleph_{0}}$. In this case we do not prescribe the endomorphism ring of $G$, but require $\operatorname{Hom}(G, X)=0$. A by now standard argument [2] and Shelah's black box show that $G$ exists satisfying all (other) conditions in (4.11). In this case $|G|=\kappa^{\aleph_{0}}=\kappa>2^{\aleph_{0}}$, and clearly $G$ serves as counterexample for the above question. Note that Problem 3 in 22] was answered in Göbel [8].

\section{Enough PRoJeCtives AND InJeCtives In COTORSION THEORIES GENERATED BY SETS OF FINITE-RANK-FREE-BY-1 GROUPS}

In this section we apply our methods concerning $G^{\prime}$-complete groups $G$ from Sections 4 and 5 for answering Problem 2 in Salce [21, p.32]. Recall the definition of (rational) cotorsion theories and of the notion of "enough projectives" from Section 2. Let $(\mathfrak{F}, \mathfrak{C})$ be a cotorsion theory. By Lemma 2.2 it has enough projectives if any free groups $A$ gives rise to $C \in \mathfrak{C}, F \in \mathfrak{F}$ and a short exact sequence

$$
0 \longrightarrow A \longrightarrow C \longrightarrow F \longrightarrow 0 \text {. }
$$

A cotorsion theory $(\mathfrak{F}, \mathfrak{C})$ is cogenerated by a set if there is a set of groups or equivalently a single group $X$ such that $\mathfrak{F}={ }^{\perp}\left(X^{\perp}\right)$ and $\mathfrak{C}=X^{\perp}$. Recall (v), the notion "cogenerated" from Section 2! Due to our knowledge of finite-rank-by-1 groups from Section 3 we will restrict ourselves in this section to an arbitrary set $\Phi$ of finite-rank-by-1 groups (which we may replace by their direct sum). We have

Main Theorem 6.1. If the cotorsion theory $(\mathfrak{F}, \mathfrak{C})$ is cogenerated by a set $\Phi$ of finite-rank-by-1 groups, then $(\mathfrak{F}, \mathfrak{C})$ has enough projectives and enough injectives.

As a corollary (part (a)), we have the indicated answer of Salce's [21] problem. Moreover we are able to deal with "quasi cotorsion" and "local cotorsion". Recall that a group $G$ is quasi cotorsion if $\operatorname{Ext}\left(\bigoplus_{p} \mathbb{Z}_{(p)}, G\right)=0$, where $p$ runs over all 
primes. Dually we define "locally cotorsion", see Salce [21. If $\mathbb{Q}^{p}$ is the subring $\left\{\frac{z}{p^{n}}: z, n \in \mathbb{Z}\right\}$ of $\mathbb{Q}$, then $G$ is locally cotorsion if $\operatorname{Ext}\left(\bigoplus_{p} \mathbb{Q}^{p}, G\right)=0$. Obviously $\bigoplus_{p} J_{p}\left(J_{p}=p\right.$-adic integers $)$ is locally cotorsion but surely not cotorsion; more details are in [21]. Note that $\Phi=\left\{\mathbb{Z}_{(p)}: p\right.$ any prime $\}$, or $\Phi=\left\{\mathbb{Q}^{p}: p\right.$ any prime $\}$, like any rational group, satisfies the hypothesis of (6.1).

Corollary 6.2. (a) All rational cotorsion theories have enough projectives and enough injectives.

(b) The quasi cotorsion and the locally cotorsion theory have enough projectives and enough injectives.

Remark. If the rational group in (a) is $\mathbb{Q}$, then Corollary (6.2) (a) is a classical result due to the founders of "cotorsion"; see D. K. Harrison and details in Fuchs [6].

Proof of (6.1). By Salce's Lemma 2.2 it is enough to begin with a free abelian group $A$ and to construct $A^{\prime} \supset A$ such that

(i) $\operatorname{Ext}\left(G^{\prime}, A^{\prime}\right)=0$ for all $G^{\prime} \in \Phi$, and if $F=A^{\prime} / A$ also

(ii) $F \in \mathfrak{F}_{\Phi}$,

or equivalently

$$
F \in{ }^{\perp}(\Phi)^{\perp}, \text { that is, }(\Phi \perp X \Rightarrow F \perp X),
$$

or more explicitly

$$
\left(\operatorname{Ext}\left(G^{\prime}, X\right)=0 \text { for all } G^{\prime} \in \Phi\right) \Rightarrow \operatorname{Ext}(F, X)=0 .
$$

We begin with $(i)$, the construction of $A^{\prime}$. By Theorem 4.8 we can find an extension $A^{\prime}=\bigcup_{\alpha<\lambda} A_{\alpha}^{\prime}$ with $A_{0}^{\prime}=0$ and $A_{1}^{\prime}=A$ such that $A^{\prime}$ is $\Phi$-complete.

Hence $\operatorname{Ext}\left(G^{\prime}, A^{\prime}\right)=0$ for all $G^{\prime} \in \Phi$, and $A^{\prime} \in \mathfrak{C}_{\Phi}$ is proved.

Now we show that

$$
0 \longrightarrow X \longrightarrow H \longrightarrow F \longrightarrow 0
$$

splits. Using the Ext-Lemma 3.3 and $\operatorname{Ext}\left(G^{\prime}, X\right)=0$ for all $G^{\prime} \in \Phi$, we have that

(iii) $\quad X$ is $G^{\prime}$-complete for all $G^{\prime} \in \Phi$.

Recall that $F=\bigcup_{\alpha<\lambda} F_{\alpha}$ with $F_{\alpha}=A_{\alpha}^{\prime} / A_{1}^{\prime}$ and $F_{1}=0$. Let $H_{\alpha}=F_{\alpha} \sigma^{-1}$. Hence $H=\bigcup_{\alpha<\lambda} H_{\alpha}$ and $H_{1}=X$, and we assume that $X \rightarrow H$ in the last exact sequence is the identity on $X$. The map $\sigma: H \rightarrow F$ in this sequence gives $\sigma_{\alpha}=\sigma \uparrow H_{\alpha}$, which induces

(iv) $H_{\alpha+1} / H_{\alpha} \cong F_{\alpha+1} / F_{\alpha}$, and inductively we will find splitting maps $\eta_{\alpha}$ such that

$(\alpha) \quad 0 \rightarrow X \rightarrow H_{\alpha} \underset{\eta_{\alpha}}{\stackrel{\sigma_{\alpha}}{\rightleftarrows}} F_{\alpha} \rightarrow 0$ and $\eta_{\beta} \subseteq \eta_{\alpha}$ for all $\beta<\alpha<\lambda$.

The desired splitting map will be $\eta=\bigcup_{\alpha<\lambda} \eta_{\alpha}$. If $\alpha=1$, then $F_{1}=0, \sigma_{1}=0$ and $\eta_{1}=0$ satisfy (1). If $\alpha$ is a limit and $(\beta)$ is defined for $\beta<\alpha$, take $\eta_{\alpha}=\bigcup_{\beta<\alpha} \eta_{\alpha}$, and $(\alpha)$ holds. It remains to construct $\eta_{\alpha}$ from $\eta_{\beta}$ for $\alpha=\beta+1$ assuming $(\beta)$. If $F_{\alpha} / F_{\beta}$ is free, then $H_{\alpha}=H_{\beta} \oplus C$ for some free abelian group $C$. In this case $\eta_{\alpha}$ is constructed easily from $\sigma_{\alpha} \mid C$. Otherwise we may assume $F_{\alpha} / F_{\beta} \cong H_{\alpha+1} / H_{\alpha} \cong G^{\prime} \in \Phi$ from (iv) and use the construction (4.8) of $A^{\prime}$. Let

$$
G^{\prime}=\left\langle y_{m}^{\prime}, x_{i}^{\prime}: i<n, m \in \omega\right\rangle
$$


with relations

$$
y_{m+1}^{\prime} p_{m}=y_{m}^{\prime}+\sum_{i<n} x_{i}^{\prime} k_{i m} \quad(m \in \omega)
$$

from (3.2). Hence we can write

$$
F_{\alpha}=\left\langle F_{\beta}, y_{m}^{\prime \prime}, x_{i}^{\prime \prime}: i<n, m \in \omega\right\rangle .
$$

The above relations turn into

(v) $y_{m+1}^{\prime \prime} p_{m}=y_{m}^{\prime \prime}+\sum_{i<n} x_{i}^{\prime \prime} k_{i m}+c_{m} \quad(m \in \omega)$ for some $c_{m} \in F_{\beta}$.

The mapping $\sigma_{\alpha}$ is surjective by $(\alpha)$, and we can find preimages $y_{m}, x_{i} \in H_{\alpha}$ and $e_{m} \in H_{\beta}$ such that $y_{m} \sigma_{\alpha}=y_{m}^{\prime \prime}, x_{i} \sigma_{\alpha}=x_{i}^{\prime \prime}$ and $e_{m} \sigma_{\beta}=c_{m}$. Note that

$$
H_{\alpha}=\left\langle H_{\beta}, y_{m}, x_{i}: i<n, m \in \omega\right\rangle
$$

by the isomorphism $(i v)$. Hence there are elements $e_{m} \in H_{\beta}$ such that the above relations become

(vi) $\quad y_{m+1} p_{m}=y_{m}+\sum_{i<n} x_{i} k_{i m}+e_{m} \quad(m \in \omega)$.

As in the proof of Theorem 5.2, we note that $\eta_{\alpha} \supset \eta_{\beta}$ can be adjusted by elements in $X=\operatorname{ker} \sigma$. We claim that $\eta_{\alpha}$ can be achieved by taking

(vii) $\quad y_{m}^{\prime \prime} \eta_{\alpha}=y_{m}+f_{m}$ and $x_{i}^{\prime \prime} \eta_{\alpha}=x_{i}+d_{i} \quad(i<n, m \in \omega)$

for some particular elements $f_{m}, d_{i} \in X$. Then $\eta_{\alpha}$ is a well-defined homomorphism if it preserves the relations $(v)$. If we apply $\eta_{\alpha}$ given by $(v i i)$ to $(v)$, we see that the relations are preserved if

(viii) $y_{m+1} p_{m}+f_{m+1} p_{m}=y_{m}+\sum_{i<n} x_{i} k_{i m}+f_{m}+\sum_{i<n} d_{i} k_{i m}+c_{m} \eta_{\beta}$

holds in $H_{\alpha}$. Put $g_{m}=-y_{m+1} p_{m}+y_{m}+\sum_{i<n} x_{i} k_{i m}+c_{m} \eta_{\beta}$, and note that $g_{m} \in$ $\operatorname{ker} \sigma=X \quad(m \in \omega)$ by the same calculation as in the proof of Theorem 5.2. By (iii) there are elements $f_{m}, d_{i} \in X$ with $f_{m+1} p_{m}=f_{m}+\sum_{i<n} d_{i} k_{i m}+g_{m}(m \in \omega)$. Again as in (5.2), the new elements ensure that (viii) holds and $\eta_{\alpha}$ exists.

\section{Notes ON SPlitters AND ON $\omega$-SPlitTers OF Size $\aleph_{1}$}

From $\S 2$ we know that splitters $<2^{\aleph_{0}}$ are $\aleph_{1}$-free. Moreover, there are obvious splitters, the free $R$-modules and torsion-free cotorsion groups - let's call them trivial splitters. There are non-trivial splitters of size $2^{\aleph_{0}}$ which are not $\aleph_{1}$-free, as shown in $\S 5$. Hence it is natural to deal with $\aleph_{1}$-free splitters of cardinality $\aleph_{1}$, a problem which became an independent topic, now separated in a joint paper [11. We mention from [11 that all $\aleph_{1}$-free splitter of size $\aleph_{1}$ are free. Particular splitters are $\omega$-splitters as defined by Schultz [22]:

Definition 7.1. A group $G$ is an $\omega$-splitter if $\operatorname{Ext}\left(\bigoplus_{\omega} G, \bigoplus_{\omega} G\right)=0$. Recall that $\bigoplus_{\omega} G=\bigoplus_{n \in \omega} e_{n} G$ is a direct sum of $\omega$ copies of $G$.

First we apply the Ext-Lemma and rederive the following result, due to Phil Schultz [22].

Proposition 7.2. If $A$ and $G$ are two torsion-free abelian groups with the same nucleus such that $\operatorname{Ext}\left(A, \bigoplus_{\omega} G\right)=0$, then $A$ is $\aleph_{1}$-free. 
Proof. Note that nuc $A=\operatorname{nuc} G=R \neq \mathbb{Q}$ by hypothesis of the proposition-in particular, $G \neq 0$. If $A$ is not $\aleph_{1}$-free, by Pontryagin's theorem there exists $G^{\prime} \subseteq_{*} A$ of minimal rank $n$ non-free, and $G^{\prime}$ is an $n$-free-by- $1 R$-module as considered in Section 3. From $\operatorname{Ext}\left(A, \bigoplus_{\omega} G\right)=0$ and $(*)$ we have $\operatorname{Ext}\left(G_{i}^{\prime}, \bigoplus_{\omega} G\right)=0$. The $R$-module $G^{\prime}$ gives rise to a representation (3.2) and the equations

(i) $\quad y_{m+1} p_{m}=y_{m}+\sum_{i<n} x_{i} k_{i m} \quad(i<n, m \in \omega)$.

The sequence $\left(p_{m}\right)_{m \in \omega}$ represents a non-trivial type of $G$, because nuc $A=$ nuc $G=R$, and by $(3.1)$. Hence $\bar{G}=G / \bigcap_{m \in \omega} G p_{m} \neq 0$, and $\operatorname{Ext}\left(G^{\prime}, \bigoplus_{\omega} \bar{G}\right)=0$, and

(ii) $D=\bigoplus_{n \in \omega} e_{n} \bar{G}=\bigoplus_{\omega} \bar{G}$ is $G^{\prime}$-complete

by our Ext-Lemma 3.3. If $c_{m} \in D(m \in \omega)$ we have solutions

(iii) $y_{m}, x_{i} \in D$ such that $y_{m+1} p_{m}=y_{m}+\sum_{i<n} x_{i} k_{i m}+c_{m}$.

In order to derive a contradiction, we choose some $0 \neq c \in \bar{G}$ and note that $\bigcap_{m \in \omega} \bar{G} p_{m}=0$. Then let $c_{n}=e_{n} c$ for all $n \in \omega$. Choose $n_{0} \in \omega$ large enough so that $x_{i} \in D^{\prime}=\bigoplus_{s<n_{0}} e_{s} \bar{G}$ for any $i<n$, and modulo $D^{\prime}$ the equations (iii) become

$$
y_{m+1} p_{m} \equiv y_{m}+c_{m}
$$

Inductively we have $y_{0} \equiv y_{n+1} q_{n}-\left(c_{0}+\sum_{i \geq 1}^{n} c_{i} q_{i-1}\right)$, where we use $q_{i}=\prod_{i=0}^{i} p_{i}$. Note that $y_{n+1} q_{n} \rightarrow 0(n \rightarrow \infty)$ in the Hausdorff topology induced by $D q_{i}$ on $D$; hence

$$
-y_{0} \equiv c_{0}+\sum_{i \geq 1}^{\infty} c_{i} q_{i-1}
$$

in the limit. The right hand side is obviously not in $D / D^{\prime}$ by our choice of the $c_{i}$ 's, while the left hand side is in the direct sum $D / D^{\prime}$ because $y_{0} \in D$, a contradiction.

Next we use the main result of [11] and apply a simple but clever reduction due to Schultz [22].

Lemma 7.3 ([22]). Any torsion-free group with nucleus $R$ has an epimorphic image of size $\leq 2^{\aleph_{0}}$ with the same nucleus.

Proof. First we note that we may assume that the $R$-module $G$ in question is reduced. Let $S$ be the set of all primes $p$ with $G p \neq G$. If $p \in S$, let $\bar{G}^{p}$ be the $p$ adic completion of $G / \bigcap_{n \in \omega} G p^{n}$, and $\eta_{p}: G \rightarrow \bar{G}^{p}$ the canonical projection followed by the embedding into $\bar{G}^{p}$. Obviously there is a projection $\pi_{p}$ of the $p$-adic module such that $\frac{1}{p}$ is not in the image of $G \eta_{p} \pi_{p}$. If $\sigma=\prod_{p \in S} \eta_{p} \pi_{p}$ then $G \sigma \subseteq \prod_{p \in S} \bar{G}^{p}$, and $G \sigma$ has the same nucleus as $G$.

Combining these observations with some known facts, we are able to extend [22] by showing the following:

Theorem $7.4\left(\mathrm{ZFC}+\nabla_{\kappa}\right)$. All $\omega$-splitters of cardinality $\leq \kappa$ are free over their nuclei.

Theorem 7.4 follows from the more general 
Lemma 7.5 $\left(\mathrm{ZFC}+\diamond_{\kappa}\right)$. If $A$ and $G$ are torsion-free abelian groups with the same nucleus $R$ with $|A| \leq \kappa$ and if $\operatorname{Ext}\left(A, \bigoplus_{\omega} G\right)=0$, then $A$ is $R$-free.

Proof. First, using (7.3), we replace $G$ above by a group of cardinality $\aleph_{1}$ with the same nucleus $R$. Hence

(i) $\operatorname{Ext}\left(A, \bigoplus_{\omega} G\right)=0$ and $|G| \leq \aleph_{1}$ without loss of generality.

The claim follows by induction on $\kappa=|A|$. If $A$ is countable, then $A$ is free by (7.2). Now we may assume that $\kappa \geq \aleph_{1}$, and Theorem 1.15 in Eklof, Mekler [4, p.353] applies for regular $\kappa$ :

If $M=\bigoplus_{\omega} G$ and $\operatorname{Ext}(A, M)=0$, then $\Gamma_{M} A=0$. Here we used the induction hypothesis that $A$ is $\kappa$-free, which follows from $(*)$. From $\Gamma_{M} A=0$ we find a cub in $\kappa$ (which we may identify with $\kappa$ ) such that $A=\bigcup_{\alpha<\kappa} A_{\alpha}$ is a $\kappa$-filtration

and $0=\operatorname{Ext}\left(A_{\alpha+1} / A_{\alpha}, M\right)=\operatorname{Ext}\left(A_{\alpha+1} / A_{\alpha}, \bigoplus_{\omega} G\right)$. Hence $A_{\alpha+1} / A_{\alpha}$ is free for all $\alpha \in \kappa$, again by the induction hypothesis, and $A$ is $R$-free. If $\kappa$ is a singular cardinal, then we recall that $R$ is hereditary, and Shelah's Singular-CompactnessTheorem applies; see e.g. [4, p. 107, Theorem 3.5]. Hence $A$ is $R$-free in this case as well.

\section{REFERENCES}

[1] T. Becker, L. Fuchs, S. Shelah, Whitehead modules over domains, Forum Mathematicum 1 (1989), 53-68. MR 90a:13017

[2] A.L.S. Corner, R. Göbel, Prescribing endomorphism algebras - A unified treatment, Proceed. London Math. Soc. (3) 50 (1985), 447-479. MR 86h:16031

[3] S. E. Dickson, A torsion theory for abelian categories, Trans. Amer. Math. Soc. 121 (1966), 223-235. MR 35:162

[4] P. Eklof, A. Mekler, Almost free modules, Set-theoretic methods, North-Holland, Amsterdam 1990. MR 92e:20001

[5] B. Franzen, R. Göbel, Presribing endomorphism algeras. The cotorsion-free case. Rend. Sem. Mat. Padova 80 (1989), 215 - 241. MR 90h:16050

[6] L. Fuchs, Infinite abelian groups - Volume 1,2, Academic Press, New York (1970, 1972) MR 41:333, MR 50:2362

[7] P. A. Griffith, A solution of the splitting mixed problem of Baer, Trans. American Math. Soc. 139 (1969), 261-269. MR 39:317

[8] R. Göbel, Abelian groups with only small cotorsion images, J. Austral. Math. Soc. (Ser. A) 50 (1991), 243-247. MR 91m:20075

[9] R. Göbel, New aspects for two classical theorems on torsion splitting, Comm. Algebra 15 (1987), 2473-2495. MR 89h:13019

[10] R. Göbel, R. Prelle, Solution of two problems on cotorsion abelian groups, Archiv der Math. 31 (1978), 423-431. MR 80f:20063

[11] R. Göbel, S. Shelah, Almost free splitters, Colloq. Math. 81 (1999), 193-221.

[12] R. Göbel, J. Trlifaj, Cotilting and a hierarchy of almost cotorsion groups, to appear in Proc. Amer. Math. Soc.

[13] J. Hausen, Automorphismen gesättigte Klassen abzählbarer abelschen Gruppen, Studies on Abelian Groups, Springer, Berlin (1968), 147-181. MR 39:5691

[14] T. Jech, Set Theory, Academic Press, New York (1978). MR 80a:03062

[15] O. Kerner, Elementary stones, Comm. Algebra, 22 (1994) 1797 - 1806. MR 95d:16017

[16] M. Prest, Model theory and modules, London Math. Soc. L.N. 130 Cambridge University Press 1988. MR 89h:034061

[17] C. M. Ringel, The braid group action on the set of exceptional sequences of a hereditary artin algebra, pp. 339 - 352 in Abelian group theory and related topics, Contemporary Math. 171, American Math. Soc., Providence, R.I. 1994. MR 95m:16006

[18] C. M. Ringel, Bricks in hereditary length categories, Resultate der Mathematik 6 (1983) 64-70. MR 85a:16035 
[19] P. Rothmaler, Purity in model theory, pp. 445 - 469 in Advances in Algebra and Model Theory, Series Algebra, Logic and Applications, Vol. 9, Gordon and Breach, Amsterdam 1997. CMP 99:12

[20] A. N. Rudakov, Helices and vector bundles, London Math. Soc. Lecture Note Ser. LNM 148. MR 91e:14002

[21] L. Salce, Cotorsion theories for abelian groups, Symposia Math. 23 (1979), 11-32. MR 81j:20078

[22] P. Schultz, Self-splitting groups, Preprint series of the University of Western Australia at Perth (1980).

[23] S. Shelah, A combinatorial theorem and endomorphism rings of abelian groups II, pp. 37 86, in Abelian groups and modules, CISM Courses and Lectures, 287, Springer, Wien 1984. MR 86i:20075

[24] L. Unger, Schur modules over wild, finite dimensional path algebras with three simple modules, Journal Pure Appl. Algebra 64 (1990) 205 - 222. MR 91i:16034

[25] T. Wakamatsu, On modules with trivial self-extensions, Journal of Algebra 114 (1988), 106114. MR 89b:16020

[26] R. Warfield, Purity and algebraic compactness for modules, Pacific J. Math. 28 (1969) 699 - 719. MR 39:4212

[27] M. Ziegler, Model theory of modules, Ann. Pure Appl. Logic 26 (1984) 149 - 213. MR 86c:03034

Fachbereich 6, Mathematik und Informatik, Universität Essen, 45117 Essen, Germany

E-mail address: R.Goebel@Uni-Essen.De

Department of Mathematics, Hebrew University, Jerusalem, Israel, and Rutgers University, New Brunswick, New Jersey

E-mail address: Shelah@math.huji.ae.il 